\title{
Indications and patterns of use for parenteral nutrition in paediatric oncology
}

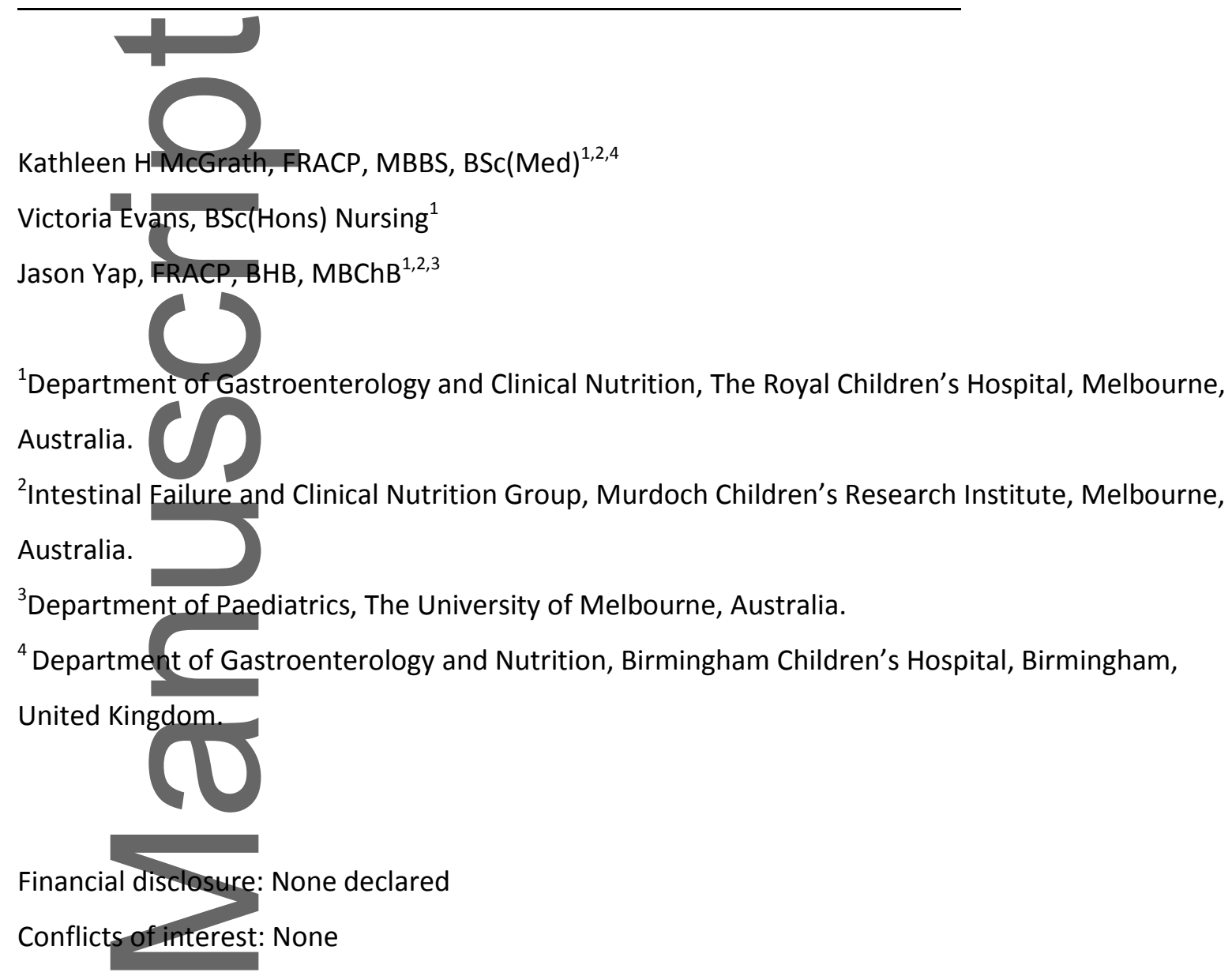

Acknowledgements: The authors would like to thank S. Andrews, J. E. Bines, E. Rogers, H. Shalley for their contributions to the study.

\section{Corresponding Author:}

Dr Kathleen H McGrath, FRACP, MBBS

Department of Gastroenterology and Clinical Nutrition

Birmingham Children's Hospital,

Steelhouse Lane, Birmingham B4 6NH, United Kingdom

Telephone: +44756 3249769

Email: mcgrath.kathleen@gmail.com

This is the author manuscript accepted for publication and has undergone full peer review but has not been through the copyediting, typesetting, pagination and proofreading process, which may lead to differences between this version and the Version of Record. Please cite this article as doi:

10.1002/jpen.1685.

This article is protected by copyright. All rights reserved. 
Background: Children with cancer are at risk of treatment related gastrointestinal toxicity and malnutrition. Intestinal dysfunction may preclude or limit use of enteral nutrition and parenteral nutrition (PN) may be required. Published indications for PN use in children with cancer are generally non-specific. We sought to describe patterns of use, indications and frequency of PN prescription in children with cancer and propose indicators to guide appropriate $\mathrm{PN}$ use.

Methods: Retrospective chart review of children with cancer receiving inpatient PN in a tertiary referral paediatric hospital over 12 months, performed using electronic medical record search and paper records. Data was summarised using descriptive statistics.

Results: PN was prescribed to 88 patients in 111 episodes. Total use (2377 days) represented $24 \%$ of annual inpatient days in all children with cancer. Primary clinical indications were mucositis (40\%), feed intolerance (29\%), post-operative (16\%) or 'other' $\rightarrow$ $(15 \%)$ which included nasogastric tube refusal $(n=6)$. Patients with haematological cancer had longer median episodes (19 vs. 12.5 days) and earlier commencement of PN after diagnosis (50 vs. 80 days) than children with non-haematological cancer. Most PN episodes (74\%) greater than 28 days were in children with Haematopoietic Stem Cell Transplant (HSCT).

Conclusion: Inpatient PN use is common in childhood cancer. Indications include mucositis, feed intolerance and surgical management. Patterns of use are influenced by cancer diagnosis and treatment type, with greater use in children with haematological cancer and HSCT. Improved understanding and specific indications may guide appropriate PN prescription, minimise unnecessary risk, cost and inconsistency in care. 


\section{Clinical Relevancy Statement:}

Parenteral nutrition (PN) use is common in children with cancer, particularly in children with<smiles>C1CC1C1CC2CC21</smiles>

Haematopoietic Stem Cell Transplant. Improved understanding of current practices in PN prescription and the associated challenges in children with cancer is important and can guide development of specific clinical tools for appropriate PN use in this high nutritional risk population. These findings are clinically relevant to avoid unnecessary risk of PN associated complications, optimise nutritional outcomes and patient experience, avoid additional cost for health services and improve consistency of patient care between services.

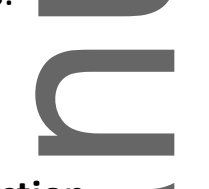

Introduction

Children with cancer are at risk of gastrointestinal toxicity related to treatment such as high dose chemotherapy, Haematopoietic Stem Cell Transplant (HSCT) or total body irradiation. The small intestine is particularly susceptible to damage and its critical role in energy and fluid homeostasis may be impaired, leading to intestinal insufficiency. Following removal or recovery from insulting agents, rapid enterocyte turnover usually allows the gastrointestinal tract to replenish itself and restore function towards normal. In many cases, reduction in gut absorptive function does not require intravenous nutritional supplementation however enteral nutrition (EN) support may be required. ${ }^{1} \mathrm{EN}$ is currently recommended as the first line nutritional intervention in children with cancer with a functional gastrointestinal tract $^{2-6}$ and has been shown to be safe, with positive outcomes for weight maintenance and reversal of malnutrition in children undergoing chemotherapy $^{2,4-6}$ or HSCT. ${ }^{7-9}$ 
Some children with cancer have severe or protracted intestinal dysfunction. Intestinal failure occurs when gut function falls below the minimum necessary for the absorption of (r) macronutrients and / or water and electrolytes and parenteral nutrition (PN) may be required to meet energy, fluid and electrolyte requirements and prevent or correct malnutrition. ${ }^{1}$ Under-recognised or persistent gastrointestinal tract damage can lead to malnutrition, prolonged hospitalisation, reduced quality of life, increased risk of infection, PN related complications and delayed chemotherapy doses. ${ }^{10-11}$ Published indications for PN in children with cancer are generally non-specific ${ }^{12-14}$ and nutrition support practices in children with cancer vary widely between institutions. ${ }^{3,4,15-18}$ This can make decision-making regarding appropriate PN use in clinical practice challenging for clinicians, especially when a dedicated nutrition support team is not available. Furthermore, clinical tools designed to assist classification of common gastrointestinal symptoms in cancer and guide nutritional $\longrightarrow$ intervention are not validated in children and their use may be limited by the subjectivity of gastrointestinal symptoms in children and challenge of elucidating symptoms in preverbal children. ${ }^{10}$

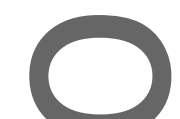

There is a need for well-planned future multicentre prospective studies comparing EN and PN use and comparing outcomes in different subgroups of children with cancer. A Cochrane review in 2015 found limited evidence to support PN as more effective for weight gain than EN in well-nourished children with cancer undergoing chemotherapy, but identified the need for more studies in this area. ${ }^{17}$ Weisdorf and colleagues reported earlier engraftment in adults and children undergoing HSCT with prophylactic PN compared with clinically indicated nutritional support but no overall benefit when other outcomes were considered and this beneficial marrow recovery effect was not supported in a subsequent study by 
Yokoyama. ${ }^{20,21}$ When compared to PN, benefits of EN include lower infection risk, less monitoring bloods required, less cost, easier administration in an outpatient setting and provision of essential trophic factors to maintain the gastrointestinal mucosa. ${ }^{2,5,14,22-26}$

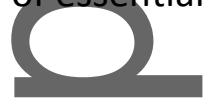

Children with cancer are a common referral group for PN however current patterns of use in this population are poorly described and published indications for PN in children with cancer are generalty non-specific. ${ }^{12-14}$ The aims of this study were to describe patterns of use, indications and frequency of PN use in children with cancer at a large tertiary paediatric referral centre with a specialised nutrition support service and to propose clinical indicators for appropriate PN use in this population.

\section{Methods}

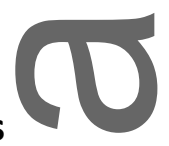

A retrospective chart review was performed of all children with a diagnosis of cancer who received inpatient PN between August 2017 and August 2018 in a large tertiary paediatric referral centre. Patients were identified using a search tool of the electronic medical record and archived PN patient lists from the nutrition support service. Patients undergoing oncological treatment e.g. HSCT but with a non-cancer primary diagnosis (e.g. primary immunodeficiency) were excluded.

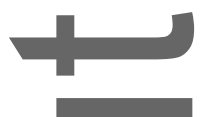

Clinical information was recorded from individual electronic medical records. Demographic details included age and gender. Clinical details recorded included cancer diagnosis, date of diagnosis, date of HSCT and / or death where applicable. Details recorded for each episode of PN use included: clinical indication, duration of episode, type of nutrient solution (standard hospital or individualised [modified electrolyte, vitamin, mineral or macronutrient 
content]), type of lipid solution used (standard lipid used in our hospital is SMOFlipid, Fresenius Kabi Australia), presence of hypertriglyceridemia (defined as triglyceride level > $3 \mathrm{mmol} / \mathrm{L})^{27,28}$ or Laboratory Confirmed Bloodstream Infection (LCBI) during an episode of PN use. LCBI were further classified as central line associated bloodstream infection (CLABSI), mucosal barrier injury or common commensal according to the VICNISS CLABSI surveillance module based on the US CDC National Healthcare Safety Network (NHSN) patient safety componentmodule. ${ }^{29}$

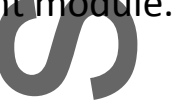

Clinical indications for PN use were recorded from the medical record. Feed intolerance was defined as severe vomiting and/or diarrhoea limiting the ability to grade up oral or enteral intake to meet estimated energy and fluid requirements. Appropriateness was assessed based on European Society of Paediatric Gastroenterology, Hepatology and Nutrition (ESPGHAN) guidelines. ${ }^{27}$ Other clinical information recorded included use of EN and presence of recent weight loss at the time of referral for PN.

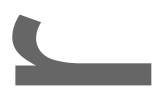

Data were summarised using descriptive statistics and analysed by underlying diagnosis (haematological versus non-haematological cancer) and presence of HSCT (HSCT versus nonHSCT). Quantitative variables were presented as mean, median and range. All categorical variables were described by absolute and percentage frequencies.

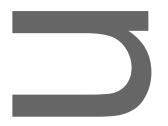

The study received approval from the hospital's human research ethics committee (HREC QA45729RCHM-2018-151532(v1)) which complies with the National Statement on Ethical Conduct in Human Research (2007) and all updates. 


\section{Results}

PN was prescribed to 88 patients ( $n=47$ haematological cancer) in 111 episodes. The most frequent haematological cancer diagnoses were: Acute Lymphoblastic Leukaemia $(n=20)$, Acute Myeloid Leukaemia $(n=11)$ and Lymphoma $(n=6)$. Common non-haematological cancer diagnoses $(n=41,47 \%)$ included: Neuroblastoma $(n=8)$, Wilms $(n=5)$, Osteosarcoma $(n=4)$ and central nervous system tumour $(n=4)$. Over one third of patients received a HSCT $(n=35,40 \%)$. The majority of these were allogenic transplants $(n=25)$. Table 1 summarises demographics of the study population.

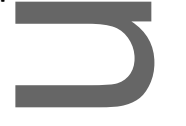

Total duration of all PN use in children with cancer over 12 months was 2377 days. The majority of patients had 1 episode of PN use $(n=69,78 \%)$ and the remainder had 2 episodes $(n=15,17 \%)$, 3 episodes $(n=3,4 \%)$ or 4 episodes $(n=1,1 \%)$. Over two third of patients with multiple episodes of PN received a HSCT (13/19, 68\%). The majority of PN episodes greater than 28 days' duration occurred in children who received HSCT $(17 / 23,74 \%)$.

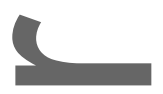

Primary clinical indications for PN use were mucositis $(n=44,40 \%)$, feed intolerance $(n=32$, $29 \%)$ and post-operative management $(n=18,16 \%)$. Remaining episodes $(n=17,15 \%)$ were attributed to 'other' indications: conservative surgical management $(n=7)$; aspiration $(n=1)$; malnutrition with patient / parent refusal of nasogastric tube (NGT) or EN ( $n=7)$ or NGT contraindicated $(n=2)$. In children with a non-haematological cancer, post-operative management was the most common clinical indication for PN use (39\%). PN indication was considered inappropriate according to ESPGHAN recommendations in $11 \%$ of episodes ( $n=12) ; n=6$ were associated with NGT refusal (all patients undergoing stem cell transplant). Six episodes had no /inadequate trial of EN. 
Table 2 and Table 3 summarise features of PN use according to the underlying cancer

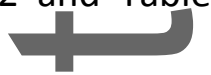

diagnosis and whether the patient received HSCT, respectively.

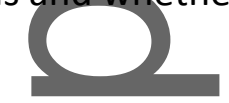

The use of individualised nutrient solution was common in children with cancer $(n=45,41 \%)$. Most reasons for individualised nutrient solution included electrolyte modifications. Almost all lipid prescriptions were for hospital standard lipid (SMOFlipid, Fresenius Kabi Australia) ( $n=109,98 \%$ and only two patients were prescribed an alternative fish-oil based lipid solution because of concern about Intestinal Failure Associated Liver Disease.

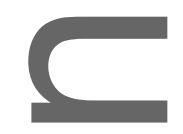

Weight loss at the time of referral for PN was common ( $n=38,34 \%)$. EN was being used at the time of PN-commencement in $49 \%$ of episodes $(n=54)$ with no identifiable trend in difference seen depending on underlying diagnosis or presence of HSCT.

Hypertriglyceridemia occurred at some point during $41 \%$ of PN episodes ( $n=46)$ and was more common in children with haematological cancer $(52 \%, n=34)$ or those with HSCT $(46 \%$, $n=24)$ than children with a non-haematological cancer $(26 \%, n=12)$ or no HSCT $(37 \%, n=22)$.

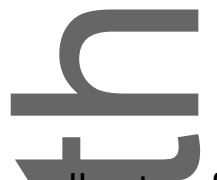

The overall rate of LCBI in children with cancer on PN was 5.0 per 1000 PN days $(n=12)$ which included mucosal barrier injury $(n=4), \operatorname{CLABSI}(n=2)$ and common commensal $(n=4)$. $\mathrm{LCBI}$ rates were higher in haematological cancer (6.8 per $1000 \mathrm{PN}$ days, $\mathrm{n}=10$ ) versus nonhaematological cancer (2.2 per $1000 \mathrm{PN}$ days, $n=2$ ). 


\section{Discussion}

Inpatient PN use is common in children with cancer and represented $24 \%$ of total inpatient (1)

days in all children with cancer (excluding day admissions) in our tertiary referral paediatric centre (internal data). PN is required by a heterogeneous group of paediatric cancer patient and the distribution of underlying cancer diagnosis for PN users in our population (53\% haematological cancer, $47 \%$ non-haematological cancer) was similar to previously published results. $^{19}$

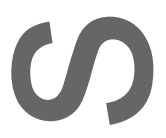

Children with haematological cancer had longer median episode duration (19 vs. 12.5 days) and earlier commencement of PN after diagnosis (50 vs. 80 days) than children with nonhaematological cancer. Earlier use may reflect gastrointestinal toxicity from high dose induction period chemotherapy regimens used for haematological cancers. The overall high<smiles>C1CCCCCCCCCCCCC1</smiles>
number of patients with non-haematological cancer who were prescribed PN was initially surprising to us. Analysis of the clinical indications revealed a large proportion of these patients (39\%) were prescribed PN as part of post-operative management (following tumour resection) which differed from the most common indicators in haematological cancer (mucositis, feed intolerance). Decisions about post-operative nutritional support can be challenging and influenced by multiple variables including challenge of predicting anticipated return of gut function, limitations associated with traditional nutritional assessment parameters e.g. weight and differing individual management approaches between surgeons. Hence, determining the appropriateness of PN prescription in some post-operative patients was difficult given the retrospective nature of this study. 
$\mathrm{PN}$ is indicated when gut function falls below the minimum necessary for the absorption of macronutrients and / or water and electrolytes and oral intake with EN is unable to sustain (ang

growth and energy requirements. ${ }^{1}$ The most common indications for PN use in children with cancer identified in our study were mucositis and feed intolerance, similar to a previous large audit of overall indications for paediatric PN use. ${ }^{19}$ However, clinical spectrum of mucositis is broad and it is important that clinicians caring for children with cancer are aware that not all patients with mucositis require PN. Patients with mild to moderate mucositis may be able to be effectively managed with oral nutrition or EN. Use of an elementary, lactose free formula with reduced long chain triglycerides provided as continuous enteral feed may maximise chances of absorption and enteral tolerance in children with cancer. ${ }^{16}$ We identified a paucity of documentation of details or grade of mucositis in the medical record, hence limiting determination of the severity of mucositis in rents most patients which was a limitation of this retrospective study. We acknowledge that clinical-classification of mucositis in the paediatric population can be challenging. Validated clinical tools from adult oncology populations are not always appropriate to be used in this group. Novel biomarkers such as citrulline may have a future role assisting classification of mucosal barrier injury severity and monitoring trends of cumulative small intestinal damage and progressive intestinal recovery in complex paediatric oncology patients. ${ }^{30-32}$

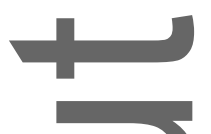

Differentiation between intestinal mucositis and feed intolerance in clinical practice can be challenging for clinicians. This study separated mucositis and feed intolerance as clinical indicators and only recorded a single primary clinical indication for each episode of PN use using available information from the medical record. However, we acknowledge that there 
may be overlap and common symptoms between the two conditions, hence potential misclassification is a limitation of our retrospective study.

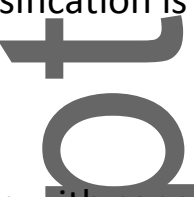

Children with cancer are exposed to many risk factors for gastrointestinal toxicity and have a high nutritional risk. Clinicians may face many challenges when determining whether PN is appropriately indicated in their patient and when it should be commenced. Current nutritional support algorithms in children with cancer are generally non-specific with respect to $\mathrm{PN}$ indications and published indications for $\mathrm{PN}$ use in general paediatric populations do not always account for the high nutritional risk of this group and its dynamic course during cancer treatment. Intestinal insults and malnutrition are frequent and supplemental use of PN may be indicated at times e.g. when significant malnutrition or weight loss exists or in the presence of gastrointestinal tract dysfunction limiting enteral tolerance. ${ }^{28}$ Using current literature available on PN use in children with cancer and the findings of this study, we propose specific clinical indications to guide appropriate prescription of $\mathrm{PN}$ in children with cancer (Figure 1). We plan to integrate these specific indicators for PN use into a clinical tool to support appropriate decision making for PN use in this population in our hospital and intend to prospectively reaudit PN use following implementation of the tool.

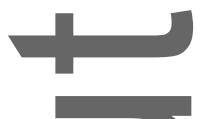

All patients $(n=6)$ prescribed PN in the context of NGT refusal and $75 \%$ of episodes of inappropriate PN use based on clinical indication (9/12) were patients receiving HSCT, reflecting challenges associated with nutrition support provision in this group. The presence of a central venous access device (CVAD) for chemotherapy may deter patients or parents from accepting nasogastric or gastrostomy insertion for EN as it is viewed as an unnecessary This article is protected by copyright. All rights reserved. 
and additional procedure when PN could be provided through an existing CVAD. Further, delayed, inadequate or inconsistent provision of information on nutrition support to parents

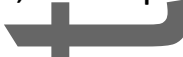
or carers of children diagnosed with cancer may also contribute to reluctance around NGT or EN and unnecessary or inappropriate PN use. ${ }^{33}$ When appropriate nutrition support clinical pathways are not in place or decision outcomes are not clear, children undergoing HSCT may easily miss 'windows of opportunity' for NGT insertion immediately pre or post HSCT before significant neutropenia and thrombocytopenia develop. Information on nutrition support options should be provided and discussed at the time of consent for HSCT and clinician response to parent or patient refusal of NGT or EN should be well thought out, informed and consistent within teams. There is a role for further research exploring clinician, parent and patient understanding of EN and PN use during HSCT and development of targeted edueation and decision aids to help standardise care between clinicians and different paediatric cancer units.

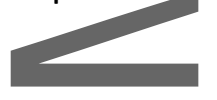

Hypertriglyceridemia was common and is influenced by critical illness, sepsis, malnutrition, medications including steroids, underlying liver disease in addition to PN. Repeated withholding of lipid in the context of high triglyceride levels with concurrent minimal oral / enteral intake or fat malabsorption may increase risk of essential fatty acid (EFA) deficiency and abnormal EFA profiles have been documented in patients undergoing $\mathrm{HSCT} .{ }^{34}$ Fat intake (2)

should be assessed for minimum recommended dosage to prevent EFA deficiency $(0.1 \mathrm{~g} / \mathrm{kg} / \mathrm{day})^{27}$ and where there is concern about insufficient intake, measurement of EFA profile and / ordasage adjustments should be considered. 
Children with cancer account for about one quarter of annual inpatient PN use in our large tertiary referral centre (internal data). PN is a costly therapy (AUD \$168 per litre per day for inpatients) when compared with other forms of nutrition therapy. Improved understanding of demand for PN use in specific groups is an important consideration in decisions about resource and budget allocations. Effective use of PN requires access to trained specialist nutrition support staff and appropriate training programs for clinicians caring for children with cancerto avoid unnecessary risks to patient safety and cost to hospitals.

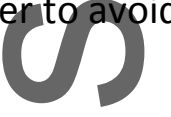

Patterns of PN use are strongly influenced by cancer diagnosis and treatment pathway with highest use in children with haematological cancer and HSCT. There is a need for wellplanned future multicentre prospective studies comparing EN and PN use in high risk groups. Appropriateness of PN use should be assessed and is important to minimise (n) unnecessary risk of complications, optimise nutritional outcomes and patient experience, avoid additional cost for health services and improve consistency of care between services.

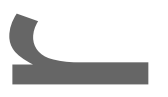

References

${ }^{1}$ Pironi L, Arends J, Baxter J, et al. ESPEN endorsed recommendations. Definition and classification of intestinal failure in adults. Clin Nutr. 2015; 34(2):171-180.

${ }^{2}$ DeSwarte-Wallace J, Firouzbakhsh S, Finklestein JZ. Using research to change practice: enteral feedings for pediatric oncology patients. J Pediatr Oncol Nurs. 2001; 18(5):217-223.

${ }^{3}$ Kuiken NSS, Rings EHHM, van den Heuvel-Eibrink MM, van de Wetering MD, Tissing WJE. Feeding strategies in pediatric cancer patients with gastrointestinal mucositis: a multicentre prospective observational study and international survey. Support Care Cancer. 2017; 25:3075-3083

${ }^{4}$ Ladas EJ, Sacks N, Meacham L, et al. A multidisciplinary review of nutrition considerations in the pediatric oncology population: a perspective from children's oncology group. Nutr Clin Pract. 2005; 20(4):377-393. 
${ }^{5}$ Pietsch JB, Ford C, Whitlock JA. Nasogastric tube feedings in children with high-risk cancer: A pilot study. J Pediatr Hematol Oncol. 1999; 21(2):111-114.

${ }^{6}$ Trimpe K, Shaw MR, Wilson M, Haberman MR. Review of the effectiveness of enteral feeding in pediatric oncology patients. J Pediatr Oncol Nurs. 2017; 34(6):439-445.

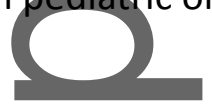

${ }^{7}$ Azarnoush S, Bruno B, Beghin L, et al. Enteral nutrition: a first option for nutritional support of childrenfollowing allo-SCT? Bone Marrow Transplant. 2012; 47(9):1191- 1195.

${ }^{8}$ Bicakli DH, Yilmaz MC, Aksoylar S, Kantar M, Cetingul N, Kansoy S. Enteral nutrition is feasible in pediatric stem cell transplantation patients. Pediatr Blood Cancer. 2012; 59(7):1327-1329

${ }^{9}$ Hastings $Y$, White $M$, Young J. Enteral nutrition and bone marrow transplantation. J Pediatr Oncol Nurs. 2006; 23(2):103-110.

${ }^{10}$ Sonis ST, Elting LS, Keefe D, et al. Perspectives on cancer therapy-induced mucosal injury: pathogenesis, mahagement, epidemiology, and consequences for patients. Cancer. 2004; 100:1995-2025.

${ }^{11}$ Elting LS, Cooksley C, Chambers M, Cantor SB, Manzullo E, Rubenstein EB. The burdens of cancer therapy. Clinical and economic outcomes of chemotherapy-induced mucositis.

Cancer. 2003; 98:1531-1539.

${ }^{12}$ Bowman LC, Williams R, Sanders M, Ringwald-Smith K, Baker D, Gajjar A. Algorithm for Nutritional Support: Experience of the metabolic and infusion support service of St. Jude Children's Research Hospital. Int J Cancer. 1998; S11:76-80.

${ }^{13}$ Sajeev M, Cohen J, Wakefield CE, Fardell JE, Cohn RJ. Decision Aid for nutrition support in Pediatric Oncology: A Pilot Study. J Parenter Enteral Nutr. 2017; 41(8):1336-1347.

${ }^{14}$ Steele C, Salazar A, Rypkema L. Utilization of a Nutrition Support Algorithm Reduces Unnecesary Parenteral Nutrition Use in Pediatric Oncology Inpatients. J Acad Nutr Diet. 2016; 116(8):1235-1238.

${ }^{15}$ Bauer J, Jurgens $\mathrm{H}$, Fruhwald MC. Important aspects of nutrition in children with cancer. Adv. Nutr. 2011; 2:67-77.

${ }^{16}$ Kuiken NSS, Rings EHHM, Tissing WJE. Risk analysis, diagnosis and management of gastrointestinal mucositis in pediatric cancer patients. Crit Rev Oncol Hematol. 2015; 94:8797.

${ }^{17}$ Ward EJ, HenryLM, Friend AJ, Wilkins S, Phillips RS. Nutritional support in children and young people with cancer undergoing chemotherapy. Cochrane Database Syst Rev. 2015 Aug 24; (8):CD003298. Doi 10.1002/14651858. 
${ }^{18}$ Mantegazza C, Landy N, Hill SM, Zuccotti GV, Kuglmeier J. Parenteral nutrition in hospitalised children. Med Clin Rev. 2016; 2(1:4):1-7.

${ }^{19}$ Common Terminology Criteria for Adverse Events (CTCAE) version 5.0. National Cancer Institute, US Department of Health and Human Services. 27 November 2017. Accessed from: https://ctep.cancer.gov/protocolDevelopment/electronic_applications/ctc.htm\#ctc_50 on 12 November 2018.

${ }^{20}$ Weisdorf S, Hofland C, Sharp HL, et al. Total parenteral nutrition in bone marrow transplantation: A clinical evaluation. J Pediatr Gastroenterol Nutr. 1984; 3:95-100.

${ }^{21}$ Yokoyama S, Fukimoto T, Mitomi T, Yabe M, Yabe H, Kato S. Use of parenteral nutrition in pediatric bone marrow transplantation. Nutrition. 1989; 5(1):27-30.

${ }^{22}$ Aquino VM, Smyrl CB, Hagg R, McHard KM, Prestridge L, Sandler ES. Enteral nutrition support by gastrostomy tube in children with cancer. J Pediatr. 1995; 127(1):58-62.

${ }^{23}$ Bisgaard Pedersen AM, Kok K, Peterson G, Nielsen OH, Michaelsen KF, Schmiegelow K. Percutaneous endoscopic gastrostomy in children with cancer. Acta Paediatr. 1999; 88:849852.

${ }^{24}$ Christensen ML, Hancock ML, Gattuso J, et al. Parenteral nutrition associated with increased infection rate in children with cancer. Cancer. 1993; 72:2732-2738.

${ }^{25}$ Guieze R, Lemal R, Cabrespine A, et al. Enteral versus parenteral nutrition support in allogenic haematopoietic stem-cell transplantation. Clin Nut. 2014; 33(3):533-538.

${ }^{26}$ Ohta K, Omura K, Hirano K, et al. The effects of an additive small amount of a low residue diet against total parenteral nutrition-induced gut mucosal barrier. Am J Surg. 2003; 185:7985.

${ }^{27}$ ESPGHAN/ESPEN/ESPR/CSPEN working group on pediatric parenteral nutrition. ESPGHAN/ESPEN/ESPR/CSPEN guidelines on pediatric parenteral nutrition. Clin Nut. 2018; 37:2306-2459.

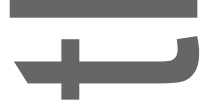

${ }^{28}$ Practical paediatric nutrition support. $2^{\text {nd }}$ Edition. Department of Gastroenterology and Clinical Nutrition, The Royal Children's Hospital. 2014.

${ }^{29}$ VICNISS CLABSI surveillance module: Central Lone-associated or Peripheral Lineassociated Bloodstream Infection (CLABSI) (PLABSI) Protocol. Victorian Healthcare Associated Infection Surveillance System (VICNISS). July 2017. Provided by Infection Control Service at The Royal Children's Hospital, Melbourne. 
${ }^{30}$ Gosselin KB, Feldman HA, Sonis AL, et al. Serum citrulline as a biomarker of gastrointestinal function during hematopoietic cell transplantation in children. J Pediatr Gastroenterol Nutr. 2014; 58(6):709-714.

ra

${ }^{31}$ Goulet 0 , Ruemmele F, Lacaille F, Colomb V. Irreversible intestinal failure. J Pediatr Gastroenterol Nutr. 2004; 38:250-269.

${ }^{32}$ Kuiken NSS, Rings EHHM, Blijlevens NMA, Tissing WJE. Biomarkers and non-invasive tests for gastrointestinal mucositis. Support Care Cancer. 2017; 25(9):2933-2941.

${ }^{33}$ Cohen J, Wakefield CE, Tapsell LC, Walton K, Cohn RJ. Parent, patient and health professional perspectives regarding enteral nutrition in paediatric oncology. Nutr Diet. 2017; 74(5):476-487.

${ }^{34}$ Clemens GW, Yamanaka W, Flournoy N, et al. Plasma fatty acid patterns of bone marrow transplant patients primarily supported by fat-free parenteral nutrition. J Parenter Enteral Nutr. 1981, 5(3):221-225.
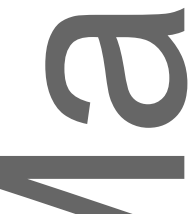

Table 1. Demographics of study population.

\begin{tabular}{|l|rrr|}
\hline \multicolumn{1}{|c|}{ Demographic feature } & $\begin{array}{r}\text { All children with } \\
\text { cancer } \\
\mathrm{n}(\%)\end{array}$ & $\begin{array}{r}\text { Haematological } \\
\text { cancer } \\
\mathrm{n}(\%)\end{array}$ & $\begin{array}{r}\text { Stem Cell } \\
\text { Transplant } \\
\mathrm{n}(\%)\end{array}$ \\
\hline $\begin{array}{l}\text { Total number of patients } \\
\text { Mean age [years] }\end{array}$ & $88(100)$ & $47(53)$ & $35(40)$ \\
Gender & 7.3 & 8.7 & 7.9 \\
& & & \\
Male & $49(56)$ & $27(57)$ & $23(71)$ \\
Fatient death & $39(44)$ & $20(43)$ & $12(34)$ \\
& $8(9)$ & $4(9)$ & $4(11)$ \\
\hline
\end{tabular}

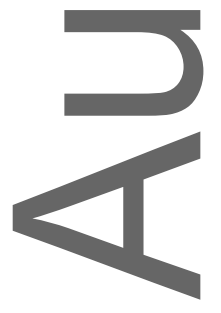


Table 2. Features of parenteral nutrition use by underlying diagnosis.

\begin{tabular}{|c|c|c|}
\hline Feature of parenteral nutrition use & $\begin{array}{r}\text { Haematological } \\
\text { cancer, } \\
\text { n [range] (\%) }\end{array}$ & $\begin{array}{r}\text { Non- } \\
\text { haematological } \\
\text { cancer, } \\
\mathrm{n} \text { [range] (\%) }\end{array}$ \\
\hline Total patien & $47(53)$ & $41(47)$ \\
\hline Total PN episodes & 65 (59) & $46(41)$ \\
\hline Mean PN episodes per patient & 1.4 [1 to 4$]$ & 1.1 [1 to 3$]$ \\
\hline Total days of PN use & 1466 & 911 \\
\hline Median days per-PN episode & 19 [3 to 108$]$ & 12.5 [2 to 133$]$ \\
\hline Less than 3 days & $0(0)$ & $1(2)$ \\
\hline 3-5 days & $9(14)$ & $6(13)$ \\
\hline 6-14 days & $12(18)$ & $19(41)$ \\
\hline $15-28$ days & $23(35)$ & $15(33)$ \\
\hline 29-90 days & $20(31)$ & $3(7)$ \\
\hline Greater than 90 days & $1(2)$ & $2(4)$ \\
\hline Median time from diagnosis to first PN episode (days) & $50[-1$ to 475$]$ & $80[-2$ to 198$]$ \\
\hline Use of standard hospital nutrient solution & $35(54)$ & $31(67)$ \\
\hline
\end{tabular}

PN, Parenteral nutrition.

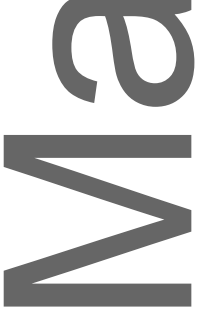

Table 3. Features of parenteral nutrition use by presence of haematopoietic stem cell transplant.

\begin{tabular}{|c|c|c|}
\hline Feature of parenteral nutrition use & $\begin{array}{r}\text { Haematopoietic } \\
\text { Stem Cell } \\
\text { Transplant, } \\
\mathrm{n} \text { [range] (\%) } \\
\end{array}$ & $\begin{array}{r}\text { No Haematopoietic } \\
\text { Stem Cell } \\
\text { Transplant, } \\
\mathrm{n} \text { [range] (\%) }\end{array}$ \\
\hline Total patien & $35(40)$ & $53(60)$ \\
\hline Total PN episode & $52(47)$ & $59(53)$ \\
\hline Mean PN episodes per patient & $1.5[1$ to 4$]$ & 1.1 [1 to 2$]$ \\
\hline Total days of PN use & 1443 & 934 \\
\hline Median days per PN episode & 24 [3 to 108$]$ & 11 [2 to 133$]$ \\
\hline Duration of PN episode & & \\
\hline Less than 3 days & $0(0)$ & $1(2)$ \\
\hline 3-5 days & $5(10)$ & $10(17)$ \\
\hline $6-14$ days & $9(17)$ & $22(37)$ \\
\hline $15-28$ days & $19(36)$ & $19(32)$ \\
\hline 29-90 days & $17(33)$ & $6(10)$ \\
\hline Greater than 90 days & $2(4)$ & $1(2)$ \\
\hline Median time from diagnosis to first PN episode (days) & 118 [15 to 475$]$ & $50[-2$ to 308$]$ \\
\hline
\end{tabular}

This article is protected by copyright. All rights reserved. 
PN episodes commencing pre-HSCT

Median start of PN commenced post-HSCT (day post HSCT)

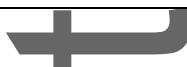

HSCT, Haematopoietic Stem Cell Transplant; PN, Parenteral nutrition.

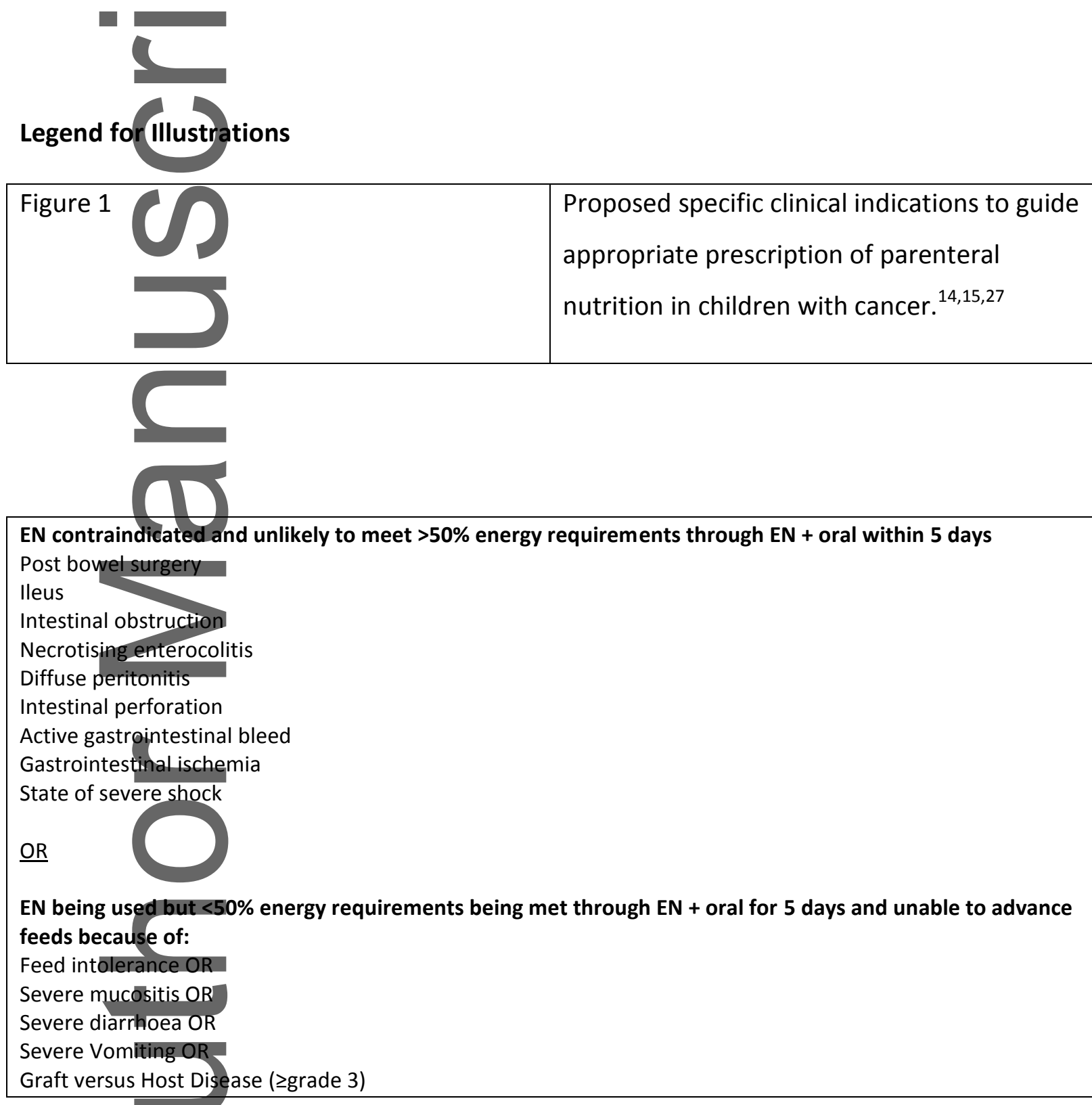

EN, Enteral nutrition.

This article is protected by copyright. All rights reserved. 


\section{University Library}

\section{- M M N E R VA A gateway to Melbourne's research publications}

Minerva Access is the Institutional Repository of The University of Melbourne

Author/s:

McGrath, KH;Evans, V;Yap, J

Title:

Indications and Patterns of Use for Parenteral Nutrition in Pediatric Oncology

Date:

2019-07-22

Citation:

McGrath, K. H., Evans, V. \& Yap, J. (2019). Indications and Patterns of Use for Parenteral

Nutrition in Pediatric Oncology. JOURNAL OF PARENTERAL AND ENTERAL NUTRITION, 44 (4), pp.632-638. https://doi.org/10.1002/jpen.1685.

Persistent Link:

http://hdl.handle.net/11343/286172 